\title{
NEW PERSPECTIVES ON RHYTHMIC CANONS AND THE SPECTRAL CONJECTURE
}

\author{
EMMANUEL AMIOT
}

\section{INTRO}

1.1. Purpose. After some impressive developments in 2003-2005, the study of rhythmic canons had slowed down, or almost stalled. Discussion of our results and ideas with specialists of more general tiling problems, also featured in this issue of the journal, opened new alleys for exploration. In particular, the quest for all Vuza canons, which is interesting both practically and theoretically, has started anew. Fresh results also suggest future directions for exploration and renew hope for solution of long standing conjectures.

1.2. Some definitions. This might be redundant with other papers in this issue, but it seemed desirable for clarity's sake to state the basic definitions.

Definition 1. For the purposes of the present paper, a rhythmic canon $(R C)$ is a tiling of the cyclic group $\mathbb{Z}_{n}$ by translations, i.e. $\mathbb{Z}_{n}$ is the disjoint union of translates of some subset $A$ :

$$
\mathbb{Z}_{n}=A \cup\left(A+b_{1}\right)+\left(A+b_{2}\right)+\cdots=A \oplus\left\{0, b_{1}, \ldots\right\}=A \oplus B
$$

$A$ is called the inner rhythm and $B$ the outer rhythm.

Musically this can be rendered as a canon (say with percussion instruments) repeating with period $n$, playing any motif (modelized as a collection of integers corresponding to the beats played) that reduces to $A$ modulo $n$, beginnning each instance of this motif on beats congruents modulo $n$ to the $b_{i}$ 's.

The algebraic notion closest to the idea of a tile in $\mathbb{Z}_{n}$ would be the orbit of $A$ under the action of $\mathbb{Z}_{n}$ by translation, i.e. the set $\left\{A+k, k \in \mathbb{Z}_{n}\right\}$. If we visualize $\mathbb{Z}_{n}$ as a circle, it means $A$ is viewed up to rotation - for a periodic event, the question of a 'starting beat' is irrelevant, though musically of course this is different. In practice, we will assume for starters that $0 \in A$, and the results will usually be stated up to such a rotation. Let it be said once and for all that it may be convenient to interpret any tile $A \subset \mathbb{Z}_{n}$ as a subset of the non negative integers, beginning with 0 and with minimal largest element:

$$
A=\left\{a_{i}, i=0 \ldots k-1\right\}, 0=a_{0}<a_{1}<\ldots a_{k-1}
$$




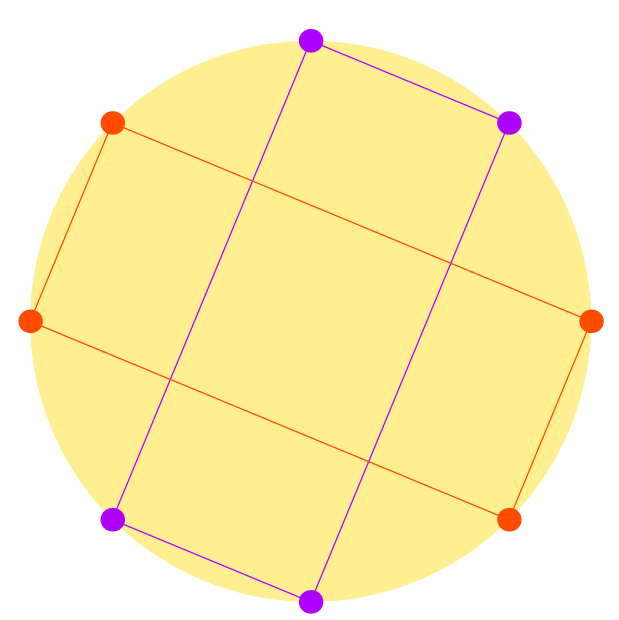

Figure 1. A tile and one of its rotated forms

Of course inner and outer rhythms can be exchanged: it is the duality of RC, see fig. 2 .
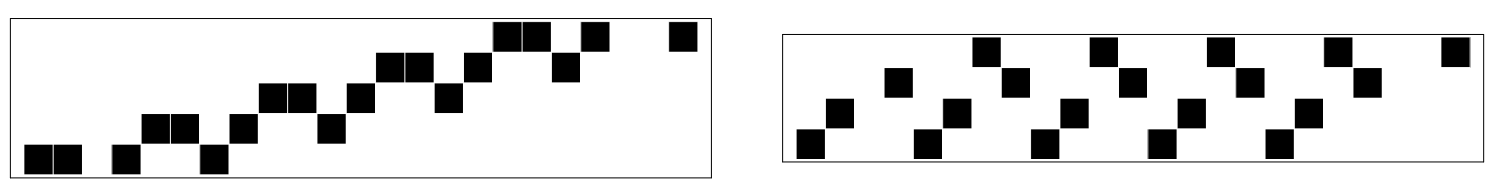

Figure 2. A canon and its dual

Definition 2. $A$ Vuza Canon (henceforth $V C$ ) is a tiling $A \oplus B=\mathbb{Z}_{n}$ wherein neither $A$ nor $B$ are periodic, i.e. $A+p \neq A$ and $B+p \neq B$ for all $1<p<n$.

Nearly all researchers have made use of the polynomial representation of a tiling:

Definition 3. The associated polynomial of a subset $A \subset \mathbb{Z}_{n}$ is $A(X)=\sum_{k \in A} X^{k}$.

It is well defined in the quotient ring $\mathbb{Z}[X] /\left(X^{n}-1\right)$ of polynomials modulo $X^{n}-1$. Moreover,

$A \oplus B=\mathbb{Z}_{n} \Longleftrightarrow A(X) \times B(X)=1+X+X^{2}+\ldots X^{n-1}=\frac{X^{n}-1}{X-1} \bmod \left(X^{n}-1\right)$ 
1.3. Combinatorial explosion. One good thing about VC is their (comparatively) limited number: for one thing, they only occur in 'bad groups', which are scarce among cyclic groups ( $n$ may be equal to $72,108,120,144,168,180 \ldots{ }^{1}$ ). Also, among the hundreds of millions (or more) of $\mathrm{RC},{ }^{2}$ in a bad group only one canon out of several millions is a VC. Still, owing to the huge number of possible RC for a given $n$, it is very important to develop effective and performant algorithms. Some recent, original ideas by Matolcsi and Kolountzakis enlarge the field of possible computations and open new horizons, both for practical and theoretical purposes. This enlargement, together with its foreseeable consequences, is the subject of the present paper.

\section{History}

2.1. The beginnings. In the 50's, Hajos, Redei, DeBruijn, Sands and others were working on the Hajos conjecture: in any tiling of a cyclic group is there necessarily one periodic factor ? They discovered a few counterexamples (first for $n=108$ ) then classified which were the 'good groups', or Hajos groups, wherein the conjecture is true, and which were the 'bad groups'. The classification was accomplished several years later by Sands, see [15]:

Theorem 1. Hajos groups, or good groups, are the cyclic groups with cardinality $n$ of the following form ( $p, q, r, s$ denote distinct prime numbers):

$$
n=p^{\alpha} \quad n=p^{\alpha} q \quad n=p^{2} q^{2} \quad n=p^{2} q r \quad n=p q r s
$$

The smallest 'bad group' is $\mathbb{Z}_{72}$.

In his monumental 10-year work [19], Dan Tudor Vuza actually rediscovered and proved the collection of results by the aforementioned mathematicians, with original methods. In the process, he also established several fundamental results well before they were spotted by the mathematical community when tiling problems gathered renewed interest in the late 90's - for instance Lemma 2.2 of [5], 'fundamental' in their own words, which states the invariance of the notion of tiling under the affine group on $\mathbb{Z}_{n}$ (see Prop. 2 below). Musically, an inner (or outer) rhythm that repeats itself within the overall period of the canon will tend to be perceived as the smaller submotive, repeated. Hence Vuza naturally introduced 'Rhythmic Canons of Maximal Category' that we will call 'Vuza Canons' or $\mathrm{VC}$ for short in this paper. He stated and proved the above theorem, providing on the way a construction (the Vuza algorithm) of several VC in any bad group. It will be seen that the concept is mathematically important, as $\mathrm{VC}$ are the atoms in the construction of all $\mathrm{RC}$ and their features are mirrored in one and every possible RC.

\footnotetext{
${ }^{1}$ This constitutes Sloane's integer sequence A102562, cf. http://www.research.att.com/ njas/sequences/.

${ }^{2}$ Harald Fripertinger established formuals for the enumeration of RC, see [8].
} 
The connection between Vuza's work on RC and Hajos' conjecture was drawn by Moreno Andreatta while working on his $\mathrm{PhD}$. He focused some interest of the musical community on the 'Rhythmic Canons of Maximal Categories' (VC), and several musicians (especially composers) began to experiment with them, particularly with transformation between rhythmic canons (RC). This allowed to understand that Vuza's algorithm, while it produced VC for all 'bad groups' (non Hajos groups), i.e. all possible values of $n$, did not reach all possible VC. The point was discussed at least as early as 2003 at the MaMuTh meeting in Zürich, or even previously: if, say $A \oplus B=\mathbb{Z}_{n}$ then by halving the tempo, and repeating motif $A$ one beat after itself, one gets a tiling of $\mathbb{Z}_{2 n}$ (read fig. 3 like a percussion score, each line standing for a different instrument playing at each black square):

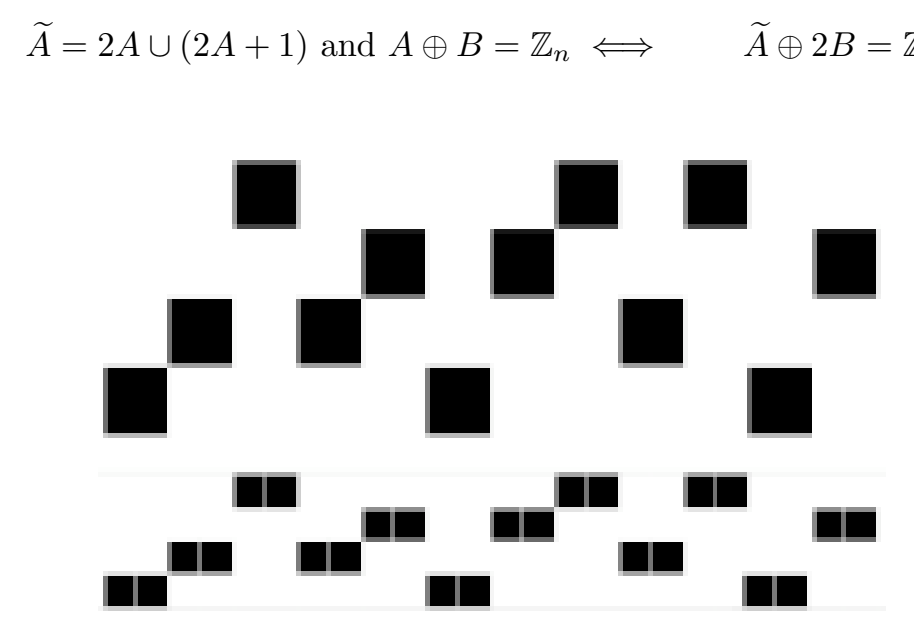

FiguRE 3. Example of 'stuttering' with a canon

In word-theory this would mean applying the morphism $0 \rightarrow 00,1 \rightarrow 11$. More to the point, the new canon is also a VC whenever the old one was. So in that way we constructed VC that were not available with the algorithms provided by Vuza (or Hajos for that matter). There was a flurry of activity in 2003-2004 when we tried all kinds of transformations in order to produce previously unchartered Vuza Canons (cf. [2]). One productive way was to look for all complements of a tile already known to be a factor of a VC, and select the aperiodic factors. This enlarged the results provided by the Vuza algorithm. Moreover, by exhaustive production of RC in a given cyclic group, Harald Fripertinger ([7]) managed to find all VC for $n=72$ and $n=108$ : as it happens, in the first case there are no others than the VC provided by Vuza's algorithm; in the latter, there are no others than the ones that we had found with complementation of previously known VC (more on this in section 2.3). 
Still the greatest breakthrough was arguably the seminal paper [5] in 1998, breaking new ground and introducing properly the sets of cyclotomic indexes of a tile (like [12] I adapt slightly the original definition, aiming at tilings of $\mathbb{Z}_{n}$ not $\left.\mathbb{Z}\right) .{ }^{3}$ Indeed, in a tiling $A \oplus B=\mathbb{Z}_{n}$, all cyclotomic polynomials ${ }^{4}$ with index $d, 1<d \mid n$, must divide $A(X)$ or $B(X)$ (sometimes both).

Definition 4. If $A \subset \mathbb{Z}_{n}$ then the sets $R_{A}=\left\{d \mid n, \Phi_{d}\right.$ is a divisor of $\left.A(X)\right\}$ and $S_{A}=$ $\left\{d \in R_{A}, d\right.$ is a prime power $\}$ are well-defined.

In particular, changing $A(X)$ by a multiple of $X^{n}-1$ does not change $R_{A}$. For instance, motif $A=\{0,1,8,9,17,28\}$ gives

$A(X)=(1+X)\left(1-X+X^{2}\right)\left(1+X+X^{2}\right)\left(1-X^{2}+X^{4}\right)\left(1-X^{3}+X^{6}\right)\left(1+X^{3}-X^{4}-X^{7}+X^{8}-X^{9}+{ }^{2}\right.$

The first factors are $\Phi_{2}, \Phi_{6}, \Phi_{3}, \Phi_{12}, \Phi_{18}$; the last one is not cyclotomic.

The pertinence of $R_{A}$ can be understood from the following:

Theorem 2. If $A \oplus B=\mathbb{Z}_{n}$ and $R_{A}=R_{A^{\prime}}$, then $A^{\prime}$ tiles with the same outer rhythm: $A^{\prime} \oplus B=\mathbb{Z}_{n}$.

This is very important in practice, as if we have a $\mathrm{RC} A \oplus B=\mathbb{Z}_{n}$ then we can look for all others $A^{\prime}$ tiling with the same $B$.

[5] establishes for the first time conditions for a motif to tile:

\section{Definition 5.}

$A \subset \mathbb{Z}_{n}$ satisfies $\left(T_{1}\right)$ if $A(1)=\prod_{p^{k} \in S_{A}} p$, the product of the prime numbers $p$ for each element $p^{\alpha}$ of $S_{A}$.

$A \subset \mathbb{Z}_{n}$ satisfies $\left(T_{2}\right)$ if for any powers of different primes $p^{\alpha}, q^{\beta}, r^{\gamma} \ldots$ in $S_{A}$, their product $p^{\alpha} \times q^{\beta}$.. lies in $R_{A}$.

They proved the following implications, the last of which is difficult:

\section{Theorem 3.}

(1) Tiling $\Rightarrow\left(T_{1}\right)$;

(2) $\left(T_{1}\right)$ and $\left(T_{2}\right) \Rightarrow$ tiling;

(3) Tiling $\mathbb{Z}_{n}$ where $n$ has at most two different prime factors $\left(n=p^{\alpha} q^{\beta}\right)$ implies $\left(T_{2}\right)$ [and still $\left(T_{1}\right)$ of course].

It is not known whether there exist tilings where the tile does not satisfy condition $\left(T_{2}\right)$. In the example above, $\left(T_{1}\right)$ reads " $A(1)=6=2 \times 3$ " and $\left(T_{2}\right)$ reduces to the same algebraic identity $2 \times 3=6$, this time stating that $6 \in R_{A}$.

\footnotetext{
${ }^{3}$ It has been known since the 50's that any tiling of $Z$ by translations of a finite tile are periodic, hence it defines a tiling of some cyclic group. See for instance $[4,1,6]$ for details.

${ }^{4}$ The $d^{\text {th }}$ cyclotomic polynomial $\Phi_{d} \in \mathbb{Z}[X]$ is an irreducible polynomial whose roots are the generators of the group of $d^{\text {th }}$ roots of unity. They can be computed recursively with the formula $\prod_{d \mid n} \Phi_{d}=X^{n}-1$.
} 
The obvious (obvious to state) " $\left(T_{2}\right)$ conjecture" is one that Coven and Meyerowitz refused to make explicitly:

$\left(\boldsymbol{T}_{2}\right)$ conjecture. If A tiles some cyclic group, then $\left(T_{2}\right)$ is true.

Remember that the second assertion is constructive: if some $A \subset \mathbb{Z}_{n}$ satisfies $\left(T_{1}\right)$ and $\left(T_{2}\right)$, then Coven and Meyerowitz gave a formula, founded on $S_{A}$, that exhibits a complement $B$ such that $A \oplus B=\mathbb{Z}_{n}$. This is featured in the 'cyclotomic rhythmic canons' module of the software Open Music. It is also the foundation of Matolcsi's method.

2.2. The spectral conjecture. The conjecture stated by Fuglede in 1974, also called Spectral Conjecture, is one of those fundamental ideas ${ }^{5}$ that link apparently unconnected domains of mathematics, here harmonic analysis and geometry. It is a simple statement:

Fuglede's Conjecture. Tiling $\Longleftrightarrow$ Spectral.

Of course some precise definitions of those termes are required. But in the context of tilings of $\mathbb{Z}_{n}$ it will be very simple to characterize. For clarity's sake I will not give the most general definition.

Definition 6. A subset $A$ of some vector space $\left(\right.$ say $\left.\mathbb{R}^{n}\right)$ is spectral iff it admits a Hilbert base of exponentials, i.e. if any map $f \in L^{2}(A)$ can be written $f(x)=\sum f_{k} \exp \left(2 i \pi \lambda_{k} . x\right)$ for some fixed family of vectors $\left(\lambda_{k}\right)_{k \in \mathbb{Z}}$ where the maps $x \mapsto \exp \left(2 i \pi \lambda_{k} . x\right)$ are mutually orthogonal.

Without the technicalities, it means that maps on $A$ admit a Fourier decomposition. A standard example: the tile $A=[0,1)$. The maps from $A$ to $C$ are naturally seen as restrictions of 1-periodic maps on $\mathbb{R}$, hence are sums of the $e^{2 i \pi n x}, n \in \mathbb{Z}$ : this is the standard Fourier decomposition.

Definition 7. A tiles $E$ by translations iff there exists a set $\mathcal{T}$ such that $A \oplus \mathcal{T}=E$, the circle around + meaning that the translates $A+t_{i}, t_{i} \in \mathcal{T}$ do not intersect each other except on sets of measure 0.

For instance $A=[0,1]$ tiles $\mathbb{R}$ with $\mathcal{T}=\mathbb{Z}$.

Fuglede himself proved his conjecture when $A$ or $\mathcal{T}$ is a group (lattice); several other special cases have been proved. But Field medalist Terence Tao made news in 2003 with a very simple idea nobody else had had before: he looked for a counterexample, and found one - in high dimension ([18]). So far, the conjecture is known to be false for both implications, in dimension greater than 3 . Dimensions 1 and 2 are essentially open problems, ${ }^{6}$ the one dimensional case of tiling is precisely the study of RC (cf. [14]).

\footnotetext{
${ }^{5}$ Like the more famous Langlands program, or Taniyama-Weil's conjecture.

${ }^{6}$ Isabella Łaba had some results when the size of the group is not much larger than the width of the tile; but as Kolountzakis has shown, this cannot be assumed in general.
} 
We are interested in tilings of $\mathbb{Z}_{n}$. Such a tiling $A \oplus B=\mathbb{Z}_{n}$ is easily uplifted to a tiling of the integers $A+(B+n \mathbb{Z})=A+C \mathbb{Z}$. Now we can turn it into a tiling of the line $\mathbb{R}$ by $\mathbb{R}=A^{\prime} \oplus C=(A=[0,1)) \oplus C$. In this setting, the condition for being spectral can be reformulated very simply (see [13]):

Proposition 1. A finite subset $A \subset \mathbb{Z}$ is spectral if there exists $\Lambda \subset[0,1)$ with $\sharp \Lambda=\sharp A$ and for all $\lambda_{k} \neq \lambda_{j} \in \Lambda, e^{2 i \pi\left(\lambda_{j}-\lambda_{k}\right)}$ is a root of the associated polynomial $A(X)$.

There are some delicate questions about the 'rationality of the spectrum': whether these roots (lying on the unit circle) are or are not roots of unity, i.e. of cyclotomic factors of $A(X) .{ }^{7}$ However, in all cases known so far, a stronger fact than the spectral conjecture is true:

Theorem 4. (Isabella Eaba) If $A$ is a finite subset of $\mathbb{Z}$ and both $\left(T_{1}\right)$ and $\left(T_{2}\right)$ are true, then $A$ is spectral.

So the $\left(T_{2}\right)$ conjecture would imply one side of the Fuglede conjecture: tiling $\Rightarrow$ spectral. All the spectral sets we know in $\mathbb{Z}$ tile, and satisfy condition $\left(T_{2}\right)$, and all known tilings are spectral too. More details and interesting results are to be found in [13].

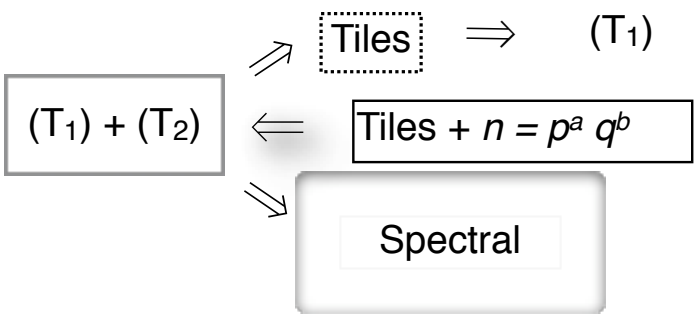

FiguRE 4. Relations between $\left(T_{1}\right),\left(T_{2}\right)$, spectral and tiling conditions

2.3. Transformations of Rhythmic Canons. Most of these transformations have been developed by composers, or at their instigation. For instance Tom Johnson (re)discovered empirically Lemma 2.2 of [5] - which is really Lemma 1.9 in [19] - e.g.

Proposition 2. If $A$ tiles $\mathbb{Z}_{n}$ with $B$ then so does $m A$ for any $m$ coprime with $n$.

In other words, the affine group mod. $n$ preserves tilings. My favorite proof relies on the fact that such transformations act on the set of roots of $A(X)$ (which is turned into $A\left(X^{m}\right)$ $\bmod X^{n}-1$ ) as an automorphism of the group of $n^{\text {th }}$ roots of unity (hence preserving their order), namely a restriction of a Galois automorphism of the cyclotomic field $\mathbb{Q}\left[e^{2 i \pi / n}\right]$.

\footnotetext{
${ }^{7}$ This must be the case whenever all roots of $A(X)$ are on or in the unit circle.
} 
This is essentially just another formulation of the irreducibility of cyclotomic polynomials, see [4]. Also it stresses that such a transformation preserves $R_{A}$, albeit only in the restricted definition (only divisors of $n$ ) that we have given. Actually I consider this feature as an argument in favour of this restriction.

This useful transformation enables

(1) to create variants of a given canon, preserving either the inner rhythm (the motif) or the outer rhythm (keeping the same motif but varying the moments when it enters). This is very satisfying musically as it offers an opportunity for variation while preserving a recognisable pattern for the listener.

(2) to classify RC in smaller orbits than under the rotation group (compare with Thm. 2 above).

For instance, there are $6 \times 3$ orbits under rotation for $\mathrm{VC}$ in $\mathbb{Z}_{72}$ but only two affine orbits for $A$ and one for $B$.

The simplest of all these transformations destroys the aperiodicity of $\mathrm{VC}$, but it is the most important:

Definition 8. The concatenation of $R C A \oplus B=\mathbb{Z}_{n}$ is obtained by concatenating the tile $A$ with itself: setting $A^{\prime}=A \oplus\{0, n, 2 n, \ldots(k-1) n\}$ one gets $A^{\prime} \oplus B=\mathbb{Z}_{k n}$.

Embedding $B$ (or even $A$ for that matter) in the larger group $\mathbb{Z}_{k n}$ can be done in several ways but the result holds anyway. More about this in $[3,4]$.

This operation enables to rephrase the definition of a VC:

Proposition 3. $A \oplus B=\mathbb{Z}_{n}$ is a VC iff neither $A \oplus B$ not $B \oplus A$ can be obtained by concatenation of a smaller canon.

Alternatively, a $\mathrm{RC}$ that is not a $\mathrm{VC}$ can be deconcatenated, i.e. reduced to a smaller $\mathrm{RC}$, and so on, recursively to either the trivial canon $\{0\} \oplus\{0\}$ or some VC.

Above I mentioned the 'stuttering operation' (see fig. 3) that turns a VC into a larger VC. This is a special case of the multiplexing operation, which is in general related to Universal Spectra ([11]) and analysed in the context of tilings of $\mathbb{Z}$ or $\mathbb{Z}_{n}$ in [4].

Proposition 4. If $A_{1}, A_{2} \ldots A_{k}$ all tile $\mathbb{Z}_{n}$ with the same outer rhythm $B$, then

$$
A=\bigcup_{i=1}^{k}\left(k A_{i}+i\right) \quad \text { tiles } \mathbb{Z}_{k n} \text { with } k B \text {. }
$$

The inverse operation is possible whenever there is equirepartition of one tile modulo some divisor of $n$; that is to say when (w.l.o.g.) the outer rhythm is divisible by some $p$. This is always the case, for instance, for $\mathrm{RC}$ in $\mathbb{Z}_{72}$. But Szabo has exhibited tilings of large cyclic groups which do not have this feature $([17])$, thus killing a few conjectures. 
The smallest known RC (VC actually) without equirepartition happen for $n=900$. Not unlike Kolountzakis' construction, Szabo starts from some very regular tiling and nudges it slightly, breaking the regularity while preserving the tiling property. This appears to be a fruitful philosophy.

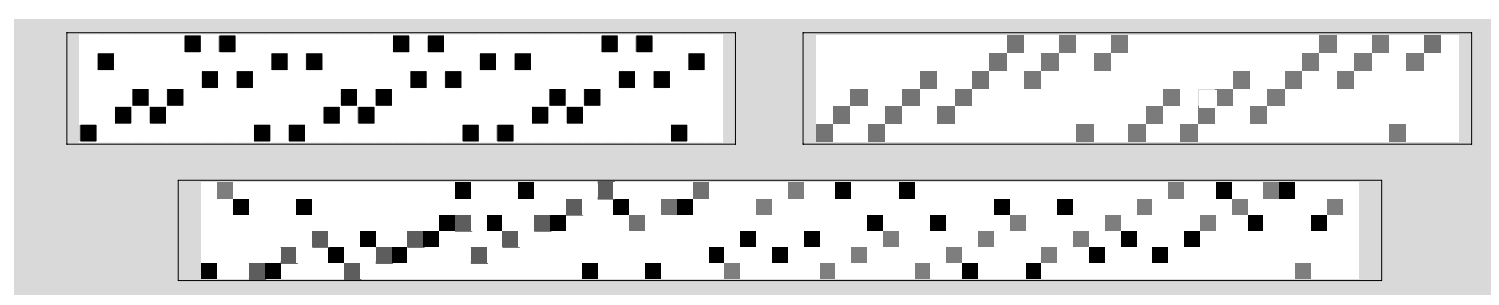

FiguRE 5. Multiplexing two canons into a Vuza Canon

2.4. Relevance to the spectral conjecture. To sum up in one sentence several partial results of mine ([3]) that were skillfully and recently completed by Edouard Gilbert [9],

Proposition 5. All the transformations in the above paragraph preserve both the spectral and the $\left(T_{2}\right)$ condition.

The consequence is straightforward: if there is a tiling with one factor that does not satisfy condition $\left(T_{2}\right)$, then either it is a Vuza or not. If not, then it is concatenated from a smaller canon, where one factor at least does not satisfy $\left(T_{2}\right)$ either. Iterating the process must end with a Vuza canon: if not, one would reduceeventually to the trivial canon $0 \oplus 0$, but it satisfies $\left(T_{2}\right)$. So

Corollary 1. The spectral and $\left(T_{2}\right)$ conjectures are true for $R C$ in general if and only they are true for $V C$.

Hence the crucial importance of Vuza canons: if we know all about VC, then we know all about all RC - notably, whether the Spectral Conjecture is true or not. Another consequence is one I stated a few years ago $([3])$ :

Corollary 2. The $\left(T_{2}\right)$ conjecture is true in any 'good group'.

This adds a few cases to the one proved by [5], like $n=p q r s$ or $n=p q r^{2}$.

\section{NEW PERSPECTIVES}

It is better to present the new techniques by way of an example, and show how they provide insight on VC. 
3.1. Example. For $n=120$, the possibilities for $S_{A}, S_{B}$ are the following:

$$
S_{A}=\{2\},\{4\},\{8\},\{3\},\{5\},\{2,3\},\{2,4\},\{2,5\},\{2,8\},\{3,4\},\{3,5\},\{3,8\},\{5,8\}
$$

and $S_{B}$ is the complement set of $S_{A}$ in $\{2,3,4,5,8\}$.

Let us consider one of the most interesting cases, $S_{A}=\{3,4\}$.

We are looking for tilings of $\mathbb{Z}_{120}$ with motif $A$, where $S_{A}$ is supposed to be equal to $\{3,4\}$. To begin with, by condition $\left(T_{1}\right) A$ has $6=\Phi_{3}(1) \times \Phi_{4}(1)$ elements; if $B$ is some outer rhythm such that $A \oplus B=\mathbb{Z}_{120}$, then $S_{B}=\{2,8,5\}$. Also, $A$ must provide a tiling of $\mathbb{Z}_{12}$, or, to be precise, the projection $A^{\prime}$ of $A$ to $\mathbb{Z}_{12}$ must tile. This is easily seen from an example (the general theorem will be addressed infra): say $A=\{0,30,32,62,88,118\}$, then $A \bmod 12=A^{\prime}=\{0,6,8,2,4,10\}$ is easily seen to tile $\mathbb{Z}_{12}$.

So one only has to start from the possible tilings of $\mathbb{Z}_{12}$ by $A^{\prime}$ 's with $S_{A^{\prime}}=S_{A}$. 12 being small, it is quickly computed. For such compliant cyclic groups, one can use the General Coven-Meyerowitz Complement formula given in [5]: it is known that for any tiling of $\mathbb{Z}_{12}$, or indeed below 120 , condition $\left(T_{2}\right)$ must be true; so the construction applies. Here for each element of $S_{B}=\{2\}$, we look for the largest factor in $n=\operatorname{lcm}\left(S_{A}\right)=12$ that is coprime with it, e.g. 3. We form $\Phi_{2}\left(X^{3}\right)=1+X^{3}$. In general, the Universal CM Complement is the product of factors obtained in this manner. Here we get $B=\{0,3\} .^{8}$ The only possibilities for $A$ tiling with $B$, up to rotation, are

$$
A_{1}^{\prime}=\{0,1,5,6,7,11\}, A_{2}^{\prime}=\{0,2,4,6,8,10\} .
$$

As we will see, they open two very different alleys, the latter giving rise to Vuza canons while the former does not. Notice that $R_{A}=\{3,4,12\}$ while $R_{A^{\prime}}=\{3,4,6,12\}$.

Solutions of $A \equiv A_{1}^{\prime} \bmod 12$ are just copies of $A_{1}^{\prime}$ with every element translated by some multiple of 12: $A=\left\{12 k_{0}, 1+12 k_{1}, 5+12 k_{2}, 6+12 k_{3}, 7+12 k_{4}, 11+12 k_{5}\right\}$. $k_{0}$ can be taken $=0$ up to rotation, it remains to compute $12^{5}=248,832$ possible $A$ 's. Among these, there are 50,000 aperiodic tiles up to rotation, like $\{0,1,5,6,7,35\}$ or $\{0,1,5,6,31,47\}$. But they come in only 6 classes of values of $R_{A}$, which are

$$
\{3,4,12\},\{3,4,12,15\},\{3,4,12,20\},\{3,4,12,20,60\},\{3,4,12,15,20,60\}
$$

Notice that 24, 40 and 120 are always missing. As it happens, this precludes any associated outer rhythm $B$ from being aperiodic. This is the trickiest part but a valuable one, as it prunes off some cases that might be long to compute. Indeed, let us consider a hypothetical outer rhythm $B$ for either of these $A$ 's. As $R_{A} \cup R_{B}=\operatorname{Div}(120)$, it means that $R_{B}$ must contain at least $2,8,5,6,24,40,120$. I pick some of those cyclotomic factors and compute their product:

$$
\begin{aligned}
\Phi_{8} \Phi_{24} \Phi_{40} \Phi_{120}=\left(X^{4}+1\right)\left(X^{8}-X^{4}+1\right)\left(X^{16}-X^{12}\right. & \left.+X^{8}-X^{4}+1\right)\left(X^{32}+X^{28}-X^{20}-X^{16}-X^{12}+X^{4}+\right. \\
& =1+X^{60} \text { must divide } B(X)
\end{aligned}
$$

${ }^{8}$ For larger numbers, a recursive construction can be envisaged. 
and this implies that $B$ is 60-periodic: for every power $X^{k}$ featuring in $B(X)$, there is also $X^{k \pm 60}$, meaning that $k \in B \Rightarrow k \pm 60 \in B$.

On the other hand, if $A \equiv A_{2}^{\prime} \bmod 12$, we get only 16,663 aperiodic solutions for $A$, with 10 possible $R_{A}$ values. Seven of those are excluded for the same reason as above, e.g. $R_{A}=\{3,4,6,12,20\}$. Others work, and indeed 18 aperiodic outer rhythms are found, e.g. $\{0,1,12,18,21,24,25,36,45,49,60,69,72,73,78,84,93,96,97,117\}$. This is more than the 8 solutions provided by Vuza's algorithm. By completion, we find that they tile with 8 different $A$ 's - exactly the 8 ones that we already knew from Vuza's algorithm.

3.2. The wheels behind the works. Several interesting features of rhythmic canons are involved in the last example. Mostly they can be found in the seminal paper [5], but they had seldom been noticed before Matolcsi made use of them. Some useful rules of thumb are taken from the forthcoming collective book on rhythmic canons to be published by Editions Delatour, Paris. Let us take them in order, begininning with the run of the mill.

Theorem 5. (1) $\sharp A=A(1)=\prod_{p^{\alpha} \in S_{A}} p . \quad\left(T_{1}\right)$

(2) If $A \oplus B=\mathbb{Z}_{n}$ then $S_{A} \cap S_{B}=\emptyset, R_{A} \cup R_{B}=\operatorname{Div}(n)$ (the divisors of $n, 1$ excepted).

Theorem 6. If $A$ tiles with some period $n$, then $A$ also tiles with period $m=\operatorname{lcm} S_{A}$, i.e. (more precisely) the projection $A^{\prime}$ of $A$ in $\mathbb{Z}_{m}$ tiles too. Also $S_{A^{\prime}}=S_{A}$.

This is musically interesting, as it enables to find a smaller period RC. I remember discussions with composer Tom Johnson, debating about the mathematical identification of motif ( $\left(\begin{array}{llll}0 & 1 & 7 & 14\end{array}\right)$ with $\left(\begin{array}{llll}0 & 1 & 2 & 3\end{array}\right)$ (modulo 4), or the essential triviality - for the mathe-

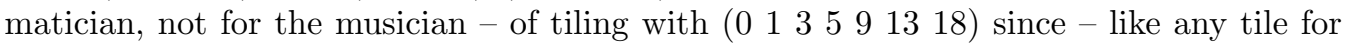
period 7 -it reduces to (0 1223456 ) modulo 7 . Interestingly enough, the best working method as in the above example consists mostly in stressing the difference between all incarnations in higher periods of a tile of a smaller group. In a way, this embodies the idea of a finer perception of complex rhythms.

Theorem 7. If A satisfies conditions $\left(T_{1}\right)$ and $\left(T_{2}\right)$, then $A$ tiles with the standard $C M$ complement $B$ which is built from $S_{A}$ alone.

The recipe for $B$ (sketched in the example above) is given in $[5,4]$ and is used for "cyclotomic canons' in the Open Music software. The interesting point is that (compare with previous theorem) only $S_{A}$ is relevant. In the same light, we have already mentioned Thm. 2, stating that sharing the same $R_{A}$ as a known tile is enough to ensure the tiling property. It actually holds when $R_{A} \subset R_{A^{\prime}}$, but not when we only assume $S_{A}=S_{A^{\prime}}$. Another interesting issue is the difficult theorem B2 of [5], completed by myself: 
Theorem 8. If $A$ tiles a $\mathbb{Z}_{n}$ with $\mathbb{Z}_{n}$ being either a 'good group' (Hajos group), OR $n$ having at most two prime factors, then $\left(T_{1}\right)$ and $\left(T_{2}\right)$ are true.

This provides a starting point for building tiles with a given $S_{A}$ in a 'good group' where the hoped-for tile is projected: this is the original idea of Matolcsi, finding the outer rhythm $B$ as a universal CM complement of the putative $A$; then completing $B$ and selecting all aperiodic $A$ 's in the list of solutions.

Now for the periodicity criteria:

Theorem 9. $A$ is periodic in $\mathbb{Z}_{n}$ (meaning $A+\tau=A$ for some $1<\tau<n$ ) iff for some maximal prime power factor $p^{\alpha}$ of $n$, all multiples of $p^{\alpha}$ are in $Z_{A}$ - or alternatively, polynomial $\frac{X^{n}-1}{X^{n / p}-1}=1+X^{n / p}+X^{2 n / p}+\ldots$ divides $A(X)$ - or again, if the Discrete Fourier Transform ${ }^{9}$ of set $A$ is nil except on a subgroup of $\mathbb{Z}_{n}$.

This enables to discard a number of cases, either because $A$ would be periodic, or because a complement (outer rhythm) $B$ would.

This is a nice feature of tiles of Vuza canons: their $R_{A}$ 's must be neither too large nor too small, but 'just right'. Though VC may appear as extreme cases in terms of canonical reduction, their aperiodicity is in fact a perfect balance, seldom achieved, between the natural propensities to periodicity of both factors $A$ and $B$.

3.3. Coming to grips. It is still mysterious how some $R_{A}$ 's are possible and some others are not. This is true in greater generality for ordinary RC: the big mystery is the gap between $S_{A}$ (which perfectly determines $S_{B}$ ) and $R_{A}$, even in the cases when condition $\left(T_{2}\right)$ is known to hold. What is one to make, for instance, of the products of elements of $S_{A}$ and $S_{B}$ ? Even condition $\left(T_{2}\right)$ tells nothing at all about these (we only have some exclusion conditions when looking for aperiodic tiles). But using the toponymy of $R_{A}$ as a starting point enables to get an inkling of which sets of divisors will give rise to canons, and which will not (see table in fig. 6). Proving such yet informal ideas (like ' $S_{A}$ must have at least two coprime elements') might significantly reduce the computation times for the search of new VC. This toponymy is a slightly broader classification than orbits under the affine group modulo $n$ (see [4]), but a much more illuminating one. We may expect shortly a few conjectures in this direction.

All but one case for $S_{A}$ had been seen before, either directly from Vuza's algorithm or by transformational techniques; but there are several new values for $R_{A}$. Also it is a huge progress to know that only the values in the table are allowed for $\mathrm{VC}$, and especially to appreciate the few values of $R_{A}$ (often only one) that are allowed for each of these $S_{A}$. For instance, it is interesting to notice how the different powers of a given prime are always intertwined between $S_{A}$ and $S_{B}$.

\footnotetext{
${ }^{9}$ Related to the associated polynomial by $F_{A}(t)=A\left(e^{2 i \pi t / n}\right)$.
} 


\begin{tabular}{|c|c|c|c|c|}
\hline $\mathbf{n}$ & $\mathbf{R}_{\mathbf{A}}$ & $\mathbf{R}_{\mathbf{B}}$ & number of A's & number of B's \\
\hline 72 & $\{2,8,9,18,72\}$ & $\{3,4,6,12,24,36\}$ & 6 & 3 \\
\hline 108 & $\{3,4,12,27,108\}$ & $\{2,6,9,18,36,54\}$ & 252 & 3 \\
\hline 120 & $\{2,3,6,8,15,24,30,120\}$ & $\{4,5,10,12,20,40,60\}$ & 20 & 16 \\
\hline 120 & $\{2,5,8,10,15,30,40,120$ & $\{3,4,6,12,20,24,60\}$ & 18 & 8 \\
\hline 144 & $\begin{array}{c}\{2,8,9,16,18,24,72,144\} \\
\text { or }\{2,8,9,16,18,72,144\}\end{array}$ & $\{3,4,6,12,24,36,48\}$ & 36 & 6 \\
\hline 144 & $\begin{array}{c}\{2,4,9,16,18,36,144\} \text { or } \\
\{2,4,6,9,16,18,36,144\} \text { or }\end{array}$ & $\{3,6,8,12,24,36,72\}$ & 8640 & 3 \\
\hline 144 & $\begin{array}{c}\{3,4,6,8,12,24,48,72\} \text { or } \\
\{3,4,6,8,12,24,36,48,72\}\end{array}$ & $\{2,9,16,18,44\}$ or & 156 & 48 \\
\hline 144 & $\begin{array}{c}\{2,3,6,8,12,24,48,72\} \text { or } \\
\{2,3,6,8,12,18,24,48,72\}\end{array}$ & $\{4,9,16,18,36,144\}$ & 324 & +12 \\
\hline
\end{tabular}

Figure 6. Classification of all Vuza canons up to $n=144$

\section{Comparison With PREvious algorithms For VC}

4.1. Completion. One strange thing about the completion routine devised by Matolcsi is that, like the Quicksort algorithm $([16]$ ), its complexity (running time) is not precisely predictable. In practice, and for heuristic reasons, it works fairly quickly, especially when the tile to complete is 'irregular' as it forces several 'no choice' situations instead of a general combinatorial explosion. But for general cases, it fares no better than the bruteforce-tree-search used by Fripertinger. It must be mentioned that Fripertinger enhanced his algorithm with a trick or two, like assuming w.l.o.g. that the first two beats in the pattern exhibit the largest gap, i.e. the program searches for $B=\left\{0=b_{0}<\cdots<b_{k}\right\}$ completing $A$ with the condition that $\forall i, b_{i}-b_{i-1} \geq b_{1}-b_{0}$. Hence the number of cases studied is not worse than $\left(\frac{n}{\sharp B}\right)^{\sharp B}$, which is lower than $e^{\sharp B}$ and often several orders of 
magnitude below that. No such formula is known for Matolcsi's algorithm, though it runs spectacularly faster in some cases, and just as slowly in others (like 'metronomes', e.g. $A=\{0, k, 2 k, 3 k, \ldots\})$. It is thus particularly well-suited to VCs.

4.2. Going further. Fripertinger's exhaustive search had left us at $n=108$ ( $n=120$ would probably have been manageable). With some ingenuity and tricks, which where developed in order to face the trickier cases, $n=144$ was completely and rather quickly solved by Matolcsi and Kolountzakis (see [12] in the same issue), and I followed with the case $n=120$ - apparently easier, but complicated by the non assumption of condition $\left(T_{2}\right)$ - shortly thereafter. I also used Matolcsi's method for checking Fripertinger's results for $n=72$, thus getting the hang of it. All in all, the computer time (once all the good moves are found) does not exceed a few hours on a personal computer - less than one hour for confirmation of the $n=72$ case. With a few improvements, all Vuza canons up to $n=288$ or maybe even 300 should be attained soon.

Alternatively, the nice 3D algorithm devised by Kolountzakis, apart from its theoretical consequences ([12]), actually enables to compute VC for reasonable values of $n$ (in IRCAM we managed to produce $\mathrm{VC}$ in $\mathbb{Z}_{180}$ with his method, though they appear to be in the previously known cyclotomic class, $\left.S_{A}=\{2,3\}\right)$. This might offer an alternative for medium-sized values of $n$ to the previously known algorithms, if it yields new solutions, and hence wider possibilities for composers. It is particularly well-suited to the computation of random VC by musical software.

4.3. Conjectures. The computations above do not address the question of whether a given subset of $\mathbb{Z}_{n}$ is, or is not, spectral. But the consideration of $R_{A}$ is directly connected with the slightly stronger ' $\left(T_{2}\right)$ conjecture'. This is known to be true in $\mathbb{Z}_{n}$ when $n$ has at most two prime factors $([5])$ and in all Hajos groups ([3]). Here the computation for all specific values of $R_{A}$ enables to focus on the cases where a counterexample might be found: factors of $S_{A}$ and $S_{B}$ have to be exactly right for it to be simply possible. ${ }^{10}$ In such a light, it might be possible to find counterexamples by researching some very specific values of $S_{A} \ldots$ at any rate if the conjecture is false. It could be a safe bet, as all settled conjectures on this subject have been wrong so far.

In the same direction but with a dual approach, it is interesting to enlarge the database of cyclic groups where ' $\left(T_{2}\right) \Longleftrightarrow$ tiling' is known to be true, $n=120$ being a first step in that direction. One good reason for this is that it enables to build larger VC recursively, using tilings of smaller groups like it was done in the example: if the $\left(T_{2}\right)$ conjecture is known to be true in the smaller group, then the 'Universal CM Complement' can be used there, speeding up the process. This is useful both for exhaustive catalogues of Vuza canons, and for trying to build counterexamples.

\footnotetext{
${ }^{10}$ For instance, for $n=120$ most partitions in $S_{A}, S_{B}$ yield factors that are known to satisfy $\left(T_{2}\right)$. An exception is $S_{A}=\{3,5,8\}$ : it took extra care to check that this case yields no Vuza Canons, starting from the other side $S_{B}=\{2,4\}$. Luckily, it also made for a shorter computation.
} 
Finally, though the Universal CM Complement is only supposed to work when $\left(T_{2}\right)$ is satisfied, paradoxically it might be the best way to construct counterexamples to the $\left(T_{2}\right)$ conjecture, as such complements $B$ for a given $S_{A}$ are by construction overloaded with superfluous cyclotomic factors; hence it may be hoped that some complements $A$ of the complement $B$ will lack one or two products of elements of $S_{A}$ in their $R_{A}$, i.e. $\left(T_{2}\right)$ might be false though $A \oplus B=\mathbb{Z}_{n}$. Moduli below 900 are unlikely to be productive in that respect, so we will need finer programs, or faster computers ${ }^{11}$ but using this wealth of new ideas, this now seems well within reach.

\section{REFERENCES}

[1] Amiot, E., Why Rhythmic Canons are Interesting, in: E. Lluis-Puebla, G. Mazzola et T. Noll (eds.), Perspectives of Mathematical and Computer-Aided Music Theory, EpOs, 190-209, Universität Osnabrück (2004).

[2] Amiot, E., Some new canons, in seminar MaMuX, January 2004, online at http://canonsrythmiques.free.fr/someNewCanons.pdf.

[3] Amiot, E., Rhythmic canons and Galois theory, Grazer Math. Ber., 347 1-25 (2005).

[4] Amiot, E., À propos des canons rythmiques, Gazette des Mathématiciens, 106 (2005).

[5] Coven, E., and Meyerowitz, A. Tiling the integers with one finite set, in: J. Alg., 212:161-174, (1999).

[6] DeBruijn, N.G., On Number Systems, Nieuw. Arch. Wisk. (3) 4, 15-17 (1956).

[7] Fripertinger, H. Remarks on Rhythmical Canons, Grazer Math. Ber., 347, 55-68 (2005).

[8] Fripertinger, H. Tiling problems in music theory, in: E. Lluis-Puebla, G. Mazzola et T. Noll (eds.), Perspectives of Mathematical and Computer-Aided Music Theory, EpOs, 149-164, Universität Osnabrück (2004).

[9] Gilbert, E., Polynô mes cyclotomiques, canons mosaï ques et rythmes k-asymé triques, mémoire de Master ATIAM, Ircam, may (2007).

[10] Hajós, G., Sur les factorisations des groupes abéliens, in: Casopsis Pest. Mat. Fys., 74:157-162 (1954).

[11] Kolountzakis, M. \& Matolcsi, M., Complex Hadamard Matrices and the spectral set conjecture, http://arxiv.org/abs/math.CA/0411512.

[12] Kolountzakis, M. \& Matolcsi, M., Algorithms for translational tiling, Journal of Mathematics and Music, 2, (2009).

[13] Laba, I., The spectral set conjecture and multiplicative properties of roots of polynomials, J. London Math. Soc. 65 , 661-671 (2002).

[14] Lagarias, J., and Wang, Y., Tiling the line with translates of one tile, in: Inv. Math., 124:341-36, (1996).

[15] Sands, A.D., The Factorization of abelian groups, Quart. J. Math. Oxford, 10(2):45-54.

[16] Sedgewick, R., Algorithms, Addison-Wesley, (2008).

[17] Szabó, S., A type of factorization of finite abelian groups, Discrete Math. 54, 121-124 (1985).

[18] Tao, T., Fuglede's conjecture is false in 5 and higher dimensions, online at http://arxiv.org/abs/math.CO/0306134.

[19] Vuza, D.T., Supplementary Sets and Regular Complementary Unending Canons, in four parts in: Canons. Perspectives of New Music, Nos 29(2) 22-49; 30(1), 184-207; 30(2), 102-125; 31(1), 270-305 (1991-1992).

\footnotetext{
${ }^{11} \mathrm{My}$ computer has been unable to give the aperiodic complements of a CM Universal Complement in $\mathbb{Z}_{900}$ in a fortnight.
} 